\title{
Design Topologies of a CMOS Charge Pump Circuit for Low Power Applications
}

\author{
Labonnah Farzana Rahman ${ }^{1, *(\mathbb{D}, \text { Mohammad Marufuzzaman }}{ }^{2}$, , Lubna Alam ${ }^{1, *}$ and Mazlin Bin Mokhtar ${ }^{1}$ \\ 1 Institute for Environment and Development, Universiti Kebangsaan Malaysia, 43600 Bangi, Malaysia; \\ mazlin@ukm.edu.my \\ 2 Institute of Energy Infrastructure, Universiti Tenaga Nasional, 43000 Selangor, Malaysia; \\ marufsust@gmail.com \\ * Correspondence: labonnah.deep@gmail.com (L.F.R.); lubna@ukm.edu.my (L.A.)
}

Citation: Rahman, L.F.;

Marufuzzaman, M.; Alam, L.;

Mokhtar, M.B. Design Topologies of a CMOS Charge Pump Circuit for Low Power Applications. Electronics 2021, 10, 676. https://doi.org/10.3390/ electronics10060676

Academic Editors: Anna Richelli and Kiat Seng Yeo

Received: 15 January 2021

Accepted: 9 February 2021

Published: 13 March 2021

Publisher's Note: MDPI stays neutral with regard to jurisdictional claims in published maps and institutional affiliations.

Copyright: (c) 2021 by the authors. Licensee MDPI, Basel, Switzerland. This article is an open access article distributed under the terms and conditions of the Creative Commons Attribution (CC BY) license (https:// creativecommons.org/licenses/by/ $4.0 /)$.

\begin{abstract}
Applications such as non-volatile memories (NVM), radio frequency identification (RFID), high voltage generators, switched capacitor circuits, operational amplifiers, voltage regulators, and DC-DC converters employ charge pump $(\mathrm{CP})$ circuits as they can generate a higher output voltage from the very low supply voltage. Besides, continuous power supply reduction, low implementation cost, and high efficiency can be managed using CP circuits in low-power applications in the complementary metal-oxide-semiconductor (CMOS) process. This study aims to figure out the most widely used CP design topologies for embedded systems on the chip (SoC). Design methods have evolved from diode-connected structures to dynamic clock voltage scaling charge pumps have been discussed in this research. Based on the different architecture, operating principles and optimization techniques with their advantages and disadvantages have compared with the final output. Researchers mainly focused on designing the charge pump topologies based on input/output voltage, pumping efficiency, power dissipation, charge transfer capability, design complexity, pumping capacitor, clock frequencies with a minimum load balance, etc. Finally, this review study summarizes with the discussion on the outline of appropriate schemes and recommendations to future researchers in selecting the most suitable $\mathrm{CP}$ design methods for low power applications.
\end{abstract}

Keywords: charge pump; topologies; CMOS; high voltage; low power

\section{Introduction}

In radio frequency identification (RFID) systems, a reader, tag and antenna are the main elements to track or identify any product information using radio-waves. RFID is a technology, which requires radio waves to track or identify any tagged object. By using the tag to any product or object allows users to track that item automatically, uniquely to track any inventory system. RFID uses readers to read the tag information attached to any entity, human, animals, fishes, products, etc. As a result, RFID-based systems are widely implemented in commercial and industrial applications for tracking product information and the supply chain management system [1-3].

A tag is typically a small chip subdivided into three main blocks: analog, logic, and memory blocks, where tag memory holds the product information data. Depending on application features, tag memory can be a read-only memory (ROM), random access memory (RAM) or electrically erasable programmable read-only memory (EEPROM), or flash memory and data buffers [4-6]. As the tag is a tiny chip, so it requires an internal power supply generator, which produces higher voltage than the power supply voltage $[2,6,7]$. Therefore, charge pump (CP) circuits are necessary for low power designs to generate the higher output voltage from a small supply voltage (VDD) in a memory chip [7-9]. Sometimes CP circuits are used as voltage doublers to generate the bias voltages in both analog and digital devices [10-12]. 
On the other hand, the charge transfer process in and out by the charge transfer capacitors is affected by transistor switches, which operated from few kilohertz to megahertz range. In non-volatile memories (NVM)-based applications, the CP circuit needed to produce higher output voltage for erasing and programming operations $[13,14]$. In this type of application, the higher voltage generated by the $\mathrm{CP}$ circuit requires voltage regulation. However, most of the research found in recent years mainly focuses on bias voltage generation function rather than a regulated voltage supply. Consequently, they deal with driving heavy loads instead of gates. In 1976, Dickson (1976) first proposed the integrated CP circuit with a static behavior model, which was again modified and further developed as a MOS diode by Witters et al. (1989), where the CP circuits dynamic behavior was described [15-20]. Previous research has been discussed with reducing power dissipation and proposed in a general way; still, there was no such literature to minimize the power dissipation, especially memory applications [21-24]. Therefore, a typical scheme to design the $\mathrm{CP}$ circuit with reduced power dissipation was independent of any specific topological implementation presented by Palumbo et al. (2002). To define the output voltage, the numbers of stages are related and the following equation defines this relation:

$$
V_{\text {out }}=N \times V_{\varnothing}+V_{\text {in }}-(N+1) \times V_{t},
$$

where $V_{\varnothing}$ is the clock amplitude, $N$ is the number of stages, $V_{i n}$ is the input supply voltage, $V_{t}$ is the threshold voltage [25]. The performance of the charge pump circuit degrades due to the body bias effect of higher pumping stages. The threshold voltage value is also increased more than two because of the higher source to bulk voltage. Moreover, some parameters such as parasitic capacitance also hold some charge and improve the $\mathrm{CP}$ circuit's internal impedance [26]. The output drive current $\mathrm{I}_{\text {out }}$ can be calculated using the following equation:

$$
I_{\text {out }}=C_{\text {Load }} \times \frac{V_{\text {out }}}{T_{\text {ramp-up }}},
$$

where $C_{\text {Load }}$ is the load capacitance, $V_{\text {out }}$ is the output voltage of the charge pump and $T_{\text {ramp-up }}$ is the time required by the charge pump to reach the output voltage [26]. On the other hand, power dissipation in $\mathrm{CP}$ circuit is another rising demand for low power applications. Whenever the voltage step value is amplified, the efficiency decreases, which is a substantial part of the circuits. The following equation gives the power consumption in the charge pump circuit,

$$
P_{D}=C \times V_{D D}^{2} \times F_{c l k},
$$

where $P_{D}$ is the power dissipation, $C$ is capacitor size and the clock frequency is $F_{c l k}$. From the equation, it is clear that a significant size capacitor and frequency can increase the output voltage but, at the same time, can increase the overall power dissipation of the $\mathrm{CP}$ circuit. Therefore, there is a significant trade-off between the performance and the $\mathrm{CP}$ circuit's power dissipation [26].

Nevertheless, research-based on minimizing the power consumption by any $\mathrm{CP}$ circuit firmly focused on simple and basic design schemes. Researchers never mentioned low voltage strategies and topologies for low power applications. As a result, the generation of higher voltage from the lower power supply voltage has been an expedition to discover energy. Initially, engineers came up with the idea of transformers that can up and down-convert the supply voltage. The auxiliary circuits to the $\mathrm{CP}$ can help progress the performance [27]. On the other hand, information or data is characterized by charge, trapped in an insulator, or inserted into the floating gate transistors through a tunnel controlled by this F-N mechanism [26].

This article will give a brief illustration of the $\mathrm{CP}$ circuit topologies design considerations. Moreover, the most widely used CP design topologies have been discussed, along with design specification and performance analysis. Finally, a summary table was plotted, illustrating the advantages and disadvantages of the CP design topologies that have intervened in the last decade with their performance comparison. Hence, this review 
article will discuss recent $\mathrm{CP}$ research trends, challenges and recommendations for future researchers to find suitable $\mathrm{CP}$ design topology for any specific design criteria, especially for low power applications.

\section{Topologies for CP Circuits}

In the last decade, $\mathrm{CP}$ design topologies have been analyzed with different design and process schemes. In this literature, some of the principal structures of $\mathrm{CP}$ circuits have been presented, based on the basic design methods of Dickson and Cockcroft and Walton's CP circuit. The increment in power supply voltage can achieve by voltage multiplication and integrating an additional one diode-capacitor voltage stage in series. In this study, different topologies, which have evolved in the last couple of years, have been discussed based on their applicability in other applications with the comparative analysis. Thus, different design schemes are classified as compatibility in-memory application, DC-DC converters and energy harvesting purposes.

\subsection{Basic CP Circuits}

Cockcroft and Walton (1932), at the Cavendish Laboratory in Cambridge, England, needed higher particle energies to accelerate sub-atomic particles along a straight discharge tube. A voltage multiplier circuit designed using a stack of capacitors connected to diodes acting as switches. By asserting and de-asserting the switches in proper sequence, they built up a potential of $800 \mathrm{kV}$ [28]. This voltage multiplier circuit was far lighter and cheaper than transformers. Cockroft and Walton designed a voltage multiplier, which converted low AC power or DC pulses to a higher DC voltage level as shown in Figure 1. In this design, capacitors were connected in series, where diodes were employed to produce high voltage.

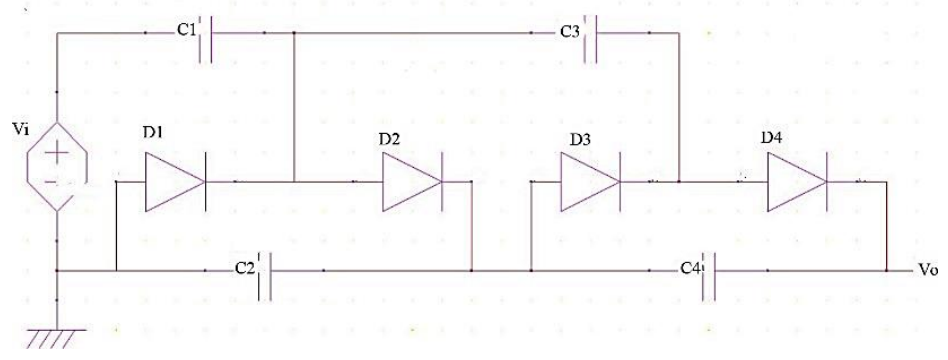

Figure 1. Schematic diagram of Cockcroft-Walton voltage multiplier [28].

Moreover, this scheme eliminated the heavy core as transformers did. In this method, the bulk of insulation/potting is required. A higher voltage was achieved from a low supply voltage using these capacitors and diodes as a multiplier. It had the advantage of low-cost materials requirement with an easy setup method [29]. However, this scheme suffered from some drawbacks; if the number of stages increased, then the higher stage's voltage started to "sag" due to capacitors' electrical impedance and the small number of steps. Moreover, when the output current is injected, a large voltage ripple is produced to increment the stages.

Dickson (1976) used the same concept as the Cockcroft-Walton voltage multiplication circuit and implemented it in IC's. It was very similar to the $\mathrm{CP}$ voltage multiplier, where the diode-connected chains are coupled to the input through several capacitors connected in parallel. Therefore, all the capacitors needed to withstand storing the full voltages produced by these chains. The system could achieve efficient multiplication by involving many capacitors. However, involving many capacitors in the circuitry increased the power dissipation and decreased pumping efficiency [16]. The primary Dickson CP circuit was very robust with different high voltage generation issues until sub-micron design technology. When the diode-connected MOSFET was turned on in the Dickson CP circuit, the threshold voltage was the voltage difference between the drain and source terminal of 
the diode-connected MOSFET. The conventional Dickson CP circuit structure had a low pumping capacity due to the large threshold voltage and the body-effect in each stage to generate the high voltages as shown in Figure 2. Additionally, all the steps suffered from a high-voltage overstress and a voltage difference, which was 2 XVDD in each node caused by each node's gate oxides. Consequently, when the number of pumping stages increases, the body effect degrades the Dickson CP circuit's pumping efficiency.

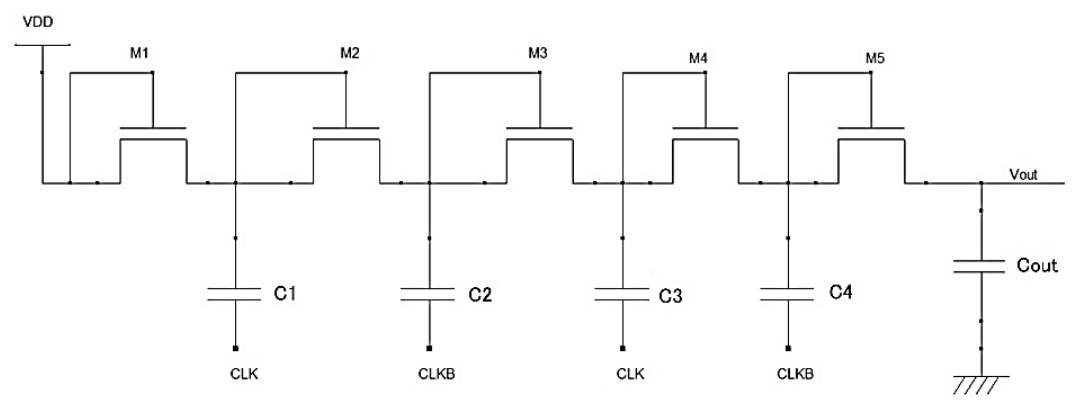

Figure 2. Dickson charge pump (CP) circuit with diode-capacitor [16].

Dickson CP circuit could not generate the desired output voltage as it was suffering from the body effect on the diode-connected MOSFETs. Therefore, Witters et al. (1989) proposed a new mechanism for designing a triple-well process $\mathrm{CP}$ [17]. In complementary metal-oxide-semiconductor (CMOS) circuit design, voltage multiplier circuits used a transistor, which performed as diodes. However, threshold voltage arises to the transistor in this circuit's chain due to the body effect problem the performance affected slightly. The output voltage level could not meet the requirements compared to a diode configuration, which eventually reduced the circuit's internal resistance. This difference between these methods got worse when any circuit implementation required more stages. Any multiplier or boosting circuit's output voltage was limited, regardless of the necessary number of steps.

Moreover, the substrate current produced in the floating-well technique affects other circuits in the same chip. Therefore, this scheme for multiplying circuit was proposed and verified for implementation. Nevertheless, this technique predicted the characteristics of a wide variety of voltage multipliers.

Atsumi et al. (1994) designed a striking and widely-implemented CP circuit named bootstrap CP. In this scheme, a simple MOS transistor or transmission gate have utilized as a switching terminal to generate higher voltage than the power supply voltage. Therefore, the MOS transistor has to switch on with a suitable supply voltage to the gate terminal, higher than the source terminal voltage. These required high gate voltages were attained from each stage to another capacitor and MOS transistor, forming the bootstrap circuit. Though the circuit generated higher output voltage, it is also clear from the complex circuit structure that it required higher implementation cost for a more complex clocking scheme and control section, which has four phases and double amplitude of the supply voltage [30,31].

\subsection{CP for Memory Application}

Lauterbach et al. (2000) illustrated a novel idea of power saving in boosted CP circuits, which doubles the power efficiency by integrating two-step adiabatic switching, charge sharing, and a simplified clocking arrangement. As this scheme shares the charges, so clock driver strengths declined. Therefore, the maximum value of the charging current dropped by a factor of three. On the other hand, combining the tri-state drivers and the clocking circuits removed the parasitic charge current peaks. As a result, the electromagnetic emission of the $\mathrm{CP}$ circuit condensed considerably. However, the charge-sharing concept necessitates this external clock generator to pump the voltage, ultimately increasing power dissipation [24]. 
Lai and Wang (2001) proposed a CP circuit where source-bulk-connected techniques were utilized with two associated courses, which solved the limitation of charge sharing methods. These circuits are involved in the primary CP circuit to reduce the body effect while avoiding the forward-biased PN junction. The body of each charge transfer transistor in the primary pumping circuit is connected to the body bias circuit. When pumping begins, the $\mathrm{CP}$ circuit draws the current from $\mathrm{CL}$ and the internal node voltage becomes lower. However, in the source-bulk connected techniques, the large bulk-to-well PN-junction capacitance increased the parasitic capacitance at each pumping node. Second, to control the diode-connected MOSFETs' body terminals dynamically, auxiliary MOSFETs are used in the source-bulk scheme, which generated the substrate current floating-well devices. However, using these extra circuitries caused large power dissipation with a large chip area [32].

Tanzawa et al. (2002) proposed a CP based on Umezawa et al.'s design principle. The proposed CP configuration required several logic circuits and PC2 switches. In this design, an additional circuit is added, which reduces the number of PC1 elements to only four. Moreover, most of the capacitors in the PC1 scheme only added a 10\% increment of the total area in the PC2 method. Additionally, in this design, only high voltage transistors are included with intrinsic capacitors [8].

Pelliconi et al. (2003) proposed a power-efficient charge pump in 2003, including low-voltage transistors and a simple two-phase clocking scheme. In this method, higher operating clocks have engaged compared to other conventional methods. As a result, it has obtained high-current, high-efficiency and small-chip area. Around $100 \mathrm{MHz}$ clock frequency has been received from the measured results. However, the circuit has secured fast switching times on low resistance and low capacitors. However, the circuit could not manage higher efficiency and boosted output voltage [33].

In 2005, Yan and Min proposed a CP method based on all P-channel metal-oxidesemiconductor (PMOS) transistors, suitable for the standard CMOS process. In this scheme, only low voltage PMOS transistors are used with the switching substrate technique to boost the transistors. Moreover, in this design, body effects have been eliminated to increase the output voltage and threshold voltage loss. This body effect was also reduced by employing the linear operation of PMOS based charge transfer switches. Therefore, this scheme has become compatible with low voltage memory applications. However, using all the PMOS transistors for transferring charge from one stage to another substrate current increased, resulting in higher power dissipation [34].

In 2008, Su and Ma proposed a four-phase CP circuit to reduce the reversion and conduction loss in each step. In this scheme, the voltage drop method is reduced in each sub-cell during the power stage. As a result, higher efficiency has been achieved with a low ripple and lower fabrication cost. Moreover, involving a subthreshold clock generator reduced the power loss in the controller. Therefore, it has provided an effective solution to the applications related to low voltage and low-power system-on-chip power converters. However, due to many capacitors, the proposed $\mathrm{CP}$ is not entirely free from parasitic effects [35].

Richelli et al. presented two CP methods for NVM with dynamic biasing of the gate and the body voltages. In this scheme, the device voltage loss problem created due to the device threshold has been controlled through gate and body biasing of every pass transistor. By using this scheme, a charge was pumped with minimal voltage drop and high conductivity. This scheme provided higher output voltage and pumping efficiency with involving only a two-phase clocking scheme. However, this design occupied a large chip area due to two pumping stages [36-38].

\section{3. $C P$ for $D C-D C$ Converter}

Shiau et al. (2007) implemented a polysilicon diode-based low voltage CP. There is no limitation of the boosted output voltage in this scheme, as polysilicon diodes are fully isolated from the silicon substrate as shown in Figure 3. Moreover, in this design method, 
$\mathrm{CP}$ is not limited by MOSFETs junction breakdown voltage, which can implement in a standard CMOS process. However, in this scheme, the body effect has not been eliminated, which is the main disadvantage of a diode-based CP circuit [39].

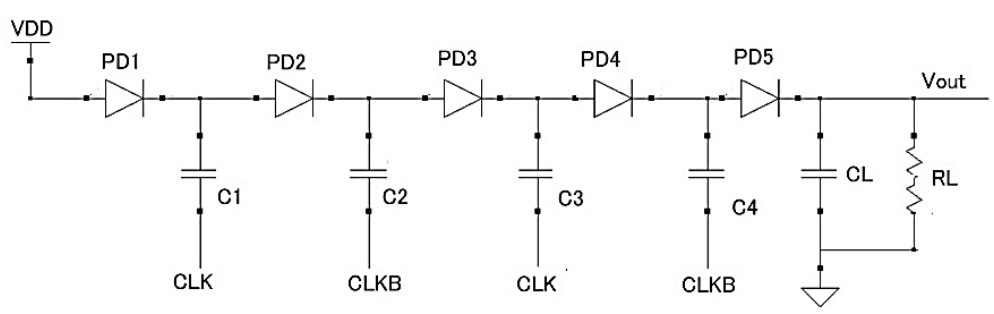

Figure 3. Static charge transfers switch based CP circuit [39].

Huang et al. proposed a CP circuit based on dual-phase mode [40]. In this scheme, several phases have been observed with the completely compact CP circuit with multiphase current-mode control. This structure involved the sensor stage, the buffer stage, the power stage, the automatic body switching (ABS) circuit, and the non-overlap circuit. The proposed structure claimed to have low ripple voltage, small chip area with high system stability. However, due to several phases, parasitic capacitance and resistance effect have not been controlled correctly in this structure.

In 2010, Peng et al. proposed a CTS-based CP circuit, where auxiliary capacitors and transistors were used to dynamically bias the CTSs to the influence of the switch's threshold voltage transistor. Moreover, this design aims to improve the pumping efficiency and lower the threshold voltage when it is turned on during charge transfer and acquires higher when turned off [41]. However, the power dissipation was high, and the pumping efficiency or voltage gain was still lower owing to the complex switching mechanism.

To remove the body effect of the diode-connected CP circuit and direct the flow of charges in pumping operation, MOSFET switches with proper on/off cycles, referred to as charge transfer switch (CTS) used to design the charge pump circuit. The CTS was able to produce better pumping gain than the diodes. Many researchers developed the CP with CTS, which required an auxiliary pass transistor to turn off the CTS entirely in the designated period [42]. Dong-Sheng et al. (2006) designed a CP circuit using the CTS topology, which had parasitic capacitors at each pumping node. The CTS method is widely used as with proper dynamic control technique of the MOSFETs to turn on/off entirely in the desired period [43].

Moreover, there was no voltage drop between the drain and source terminal. Hence, the MOSFET switch could shift the charge without suffering the constraint of the threshold voltage. The dynamic control circuit-controlled CTS to transfer the amounts from one stage to another without the threshold voltage drop [42]. However, the extra power dissipation of the parasitic capacitance caused the high-power indulgence in this scheme.

Yan et al. (2012) designed a CP circuit using an improved charge sharing method, which had six stages with six pumping capacitors at each pumping node. In this design, Yan et al. improved the charge pump circuit's performance based on the CTS method. Moreover, a zero $V_{t h}$ MOSFET was adopted to overcome the $V_{t h}$ drop at every node. Furthermore, the charge transfer switches can turn on/off completely, and feedback current reduced. However, this design dissipated an enormous power with a small number of stages due to complex circuitry [44].

Wei et al. (2013) proposed an enhanced or NCP-2 CTS CP circuit to the shortest charge flow with improved voltage pumping gain. In this design, the diode-configured design limitations were managed using a proper clock scheme and the pumping capacitors' sizing. Moreover, the strategy had the least parasitic capacitance effects. However, the system had low output voltage, higher power dissipation, and increase pumping efficiency [45].

In 2016, Zucchelli et al., proposed an inductor-based CP design scheme, fully integrated into DC/DC converter. Though the design was the modified version of the primary 
Dickson CP circuit, it provides enhanced performances due to small inductors' involvement in the first stage of the charge transfer process as shown in Figure 4a,b. Therefore, the proposed design able to reduce power dissipation, rise time and output voltage ripple. Besides, this design was free from external components, making it compatible with CMOS technology [46].

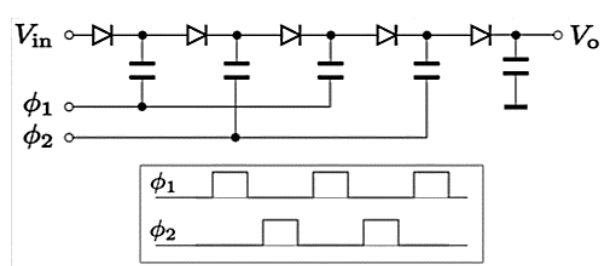

(a)

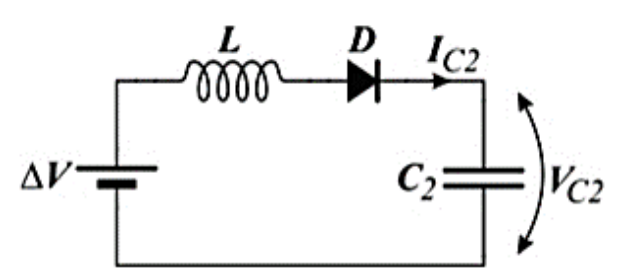

(b)

Figure 4. (a) Schematic of the inductor-based CP circuit (b) first stage of the CP [46].

Rumberg et al., in 2017, came up with a new regulated CP circuit, which has designed based on floating-gate transistors. This type of structure is suitable for applications that required tunneling voltages to program floating-gate transistors. Due to its compact architectural design, the $\mathrm{CP}$ has leveraged variable frequency regulation and minimized short-circuit current to provide stable tunneling voltages. This design can reduce power dissipation. However, this circuit is not suitable for applications that needed standard CMOS transistors [47].

A high voltage generator based on the $\mathrm{CP}$ scheme has demonstrated by Abdi et al. in 2018, which features novel voltage regulation with a variable clock frequency. In this topology, an input voltage modulation has been added to a low-dropout regulator at the input stage to forward the input voltage to the next node. As a result, higher output voltage with stable voltage regulation has been achieved in this method. Moreover, thick-oxide transistors are employed to enhance the pumping efficiency and maintain the substrate $/ \mathrm{n}$ well voltage level during operation [48].

Rahman et al. proposed a modified charge transfer switch-based CP circuit in 2020 to be compatible with RFID tag EEPROM as shown in Figure 5. In this design, instead of diode-configuration, all NMOS switches in the charge transfer nodes. As a result, this topology could manage to reduce the substrate current and overall power dissipation. Additionally, in this design, the researchers can minimize the output ripple voltage and the chip layout area. This scheme has also integrated with a voltage regulator to produce a steady-boost voltage, the RFID tag memories requirement [1].

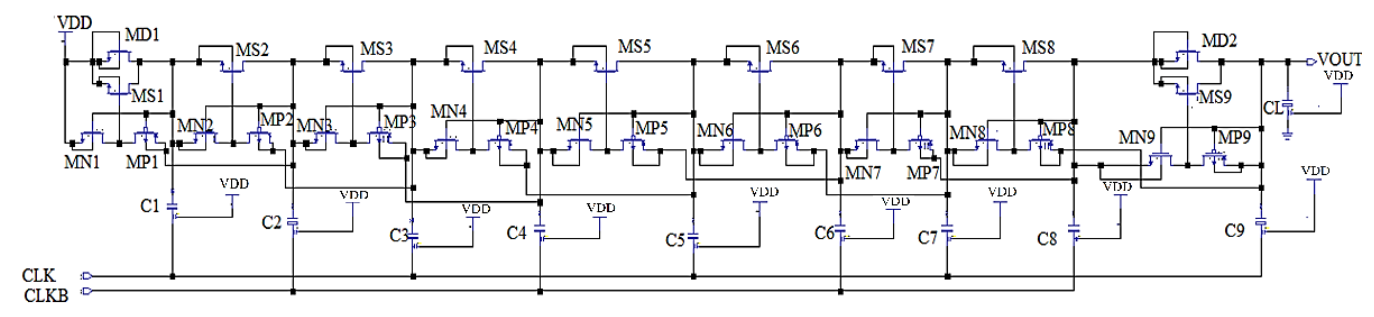

Figure 5. Schematic diagram of the CTS CP [1].

\subsection{CP for Energy Harvesting}

In 2008, Richelli et al. proposed a boosted CP design scheme suitable for energy harvesting. It has the feature of increasing output voltage from a very low supply voltage of about $150 \mathrm{mV}$. This scheme was designed using the new hybrid inductive and capacitive architecture. In particular, this method only consists of NMOS transistors, which were 
utilized as pass-transistors. Therefore, this scheme has a low threshold voltage compared to a PMOS-based CP circuit [49].

Shih and Otis et al. presented a modified four-phase CP circuit suitable for a fully integrated dc-dc converter for micropower energy harvesting. This modified $\mathrm{CP}$ can boost the voltage up to $3 \times \mathrm{VDD}$ in free-running mode even if the input voltage automatically running down up to $270 \mathrm{mV}$. To do this autonomous voltage control, this modified CP did not use any external extraction. As a result, this adjusted $\mathrm{CP}$ ensured a regulated voltage supply at $1.4 \mathrm{~V}$ with only three $\mu \mathrm{W}$ power dissipation. However, this topology was unable to provide higher pumping efficiency with a higher chip area. Simultaneously, this mechanism is not compatible with many applications as the circuit modified to be compatible with DC-DC converter for micropower energy harvesting applications with stringent size constraints [50].

Recently, Peng et al. (2014) proposed a cross-coupled-based CP circuit following the Ker et al. design [51,52].The body biasing and backward charge pump scheme was introduced in this method, which could completely turn off/on the MOS transistors. As a result, voltage loss during switching reduced and the reverse charge sharing problem decreased. Moreover, low voltage operation was arisen due to the involvement of the sub-threshold organization. Nevertheless, two branch CP circuits designed by Peng et al. resolved all the boundaries of the Ker et al. $\mathrm{CP}$ circuit, where the pumping effectiveness was found $89 \%$ and $0.1 \mathrm{mS}$ pumping speed was delivered. However, this designed $\mathrm{CP}$ required $320 \mathrm{mV}$ for start-up, which was higher than other designs with high power dissipation due to its complex circuitry. Moreover, immense leakage current occurred due to unsuitable body biasing and controlling scheme.

Kim et al. (2015) proposed a CP circuit with dynamic body-biasing (DBB) to solve the limitations of high current transfer and high body leakages as shown in Figure 6. In this scheme, dead-time restrictions, conduction loss, and constraints were taking into concern during the design process regarding high voltage adaptation efficacy (VCE) and cut the power conversion efficiency (PCE) of [53].

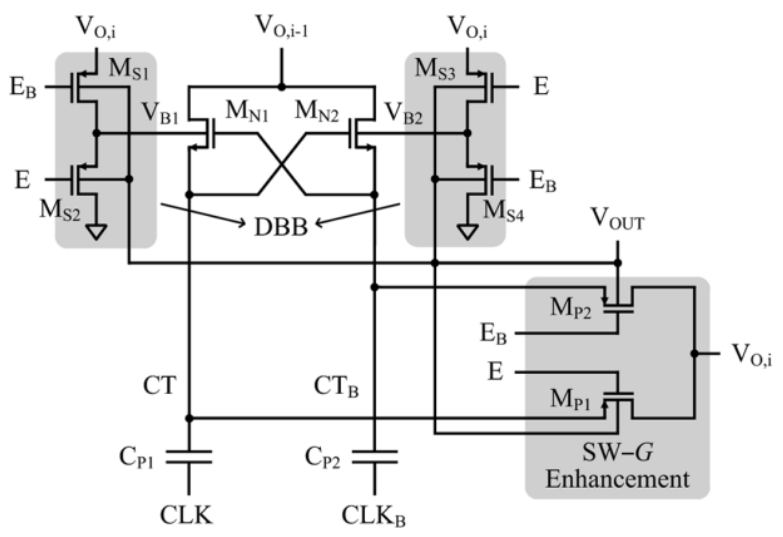

Figure 6. Dynamic-body-biasing CP [53].

In 2016, Mondal et al. proposed an inductor-less switching CP circuit suitable for solar energy harvesting. In this method, the charge transfer capability has improved and the charge sharing time to load. Moreover, in this scheme, a new single-clock treetopology has been introduced to design the $\mathrm{CP}$ circuit, which has provided better charge transfer capability and sharing time. This proposed method has produced higher pumping efficiency for applications like the microscale energy harvesting system [54].

In 2019, Li et al. proposed a temperature-insensitive CP circuit, where the output voltage included a linear adjustment range. In this method, no bipolar-CMOS-DMOS (BCD) technology has been added to generate the output voltage higher than the n-well/substrate diode's breakdown voltage, which has utilized triple-well NMOS. Besides, the pumping clock's amplitude has been controlled continuously to produce a closed-loop regulation of 
voltage to make it insensitive to temperature changes. In a closed-loop control mechanism, the $\mathrm{CP}$ output has steady voltage regulation with a voltage deviation of only $1.1 \%$. As a result, the proposed CP scheme has the compatibility of sensor accuracy for controlling micro gyroscopes. No external high voltage device must be integrated with the gyroscope measurement system [55].

\section{Discussion}

This review summarized different $\mathrm{CP}$ design methods based on their applications, as presented in Table 1. At present, low power, low ripple, higher efficiency, higher output voltage, and high-performance CP circuits are in high demand to NVMs, DCDC converters, RFID systems, PLL-based design, energy harvesting, and many other applications. During any CP circuit design process, the main concern is to ensure that the method can produce a higher output voltage from a very low supply voltage. Another critical feature of designing a CP circuit is maintaining a low power dissipation, which made it compatible with low power applications, as mentioned earlier. These applications also required a highly efficient CP design method, which could be degraded by increasing the number of CP design stages with large output ripple voltage. Additionally, a highly stable and regulated high voltage generation process is desirable to the researchers who deal with low power design specifications in CMOS.

Table 1. Performance comparison of different CP design topologies based on DC-DC converters and memory devices.

\begin{tabular}{|c|c|c|c|c|c|c|c|c|}
\hline Source & Design Topology & $\begin{array}{l}\text { Process } \\
(\mu \mathrm{m})\end{array}$ & $\begin{array}{c}\text { Supply } \\
\text { Voltage VDD } \\
\text { (V) }\end{array}$ & $\begin{array}{l}\text { Frequency } f_{c l k} \\
\quad(\mathrm{MHz})\end{array}$ & $\begin{array}{l}\text { Output } \\
V_{\text {out }}(\mathrm{V})\end{array}$ & $\begin{array}{c}\text { Pumping } \\
\text { Efficiency (\%) }\end{array}$ & $\begin{array}{l}\text { Die Area } \\
\left(\mathrm{mm}^{2}\right)\end{array}$ & Applications \\
\hline [1] & CTS & 0.13 & $1.2 \sim 1.8$ & 11.3 & $9.6 \sim 14.59$ & $88 \sim 90$ & 0.044 & $\begin{array}{l}\text { RFID tag } \\
\text { EEPROM } \\
\text { RFID }\end{array}$ \\
\hline [2] & enhanced-NCP2CTS & 0.18 & 1.8 & 20 & 5.95 & 66 & 2.4 & $\begin{array}{l}\text { transponder } \\
\text { Memory }\end{array}$ \\
\hline [8] & $\begin{array}{l}\text { intrinsic high-voltage } \\
\text { transistors }\end{array}$ & 0.18 & 1.8 & - & $4-18$ & 40 & - & $\begin{array}{c}\text { Flash } \\
\text { memories }\end{array}$ \\
\hline [24] & $\begin{array}{l}\text { two-step adiabatic } \\
\text { switching }\end{array}$ & 0.25 & 2.5 & - & 9.16 & 57 & - & $\begin{array}{c}\text { EEPROM and } \\
\text { Flash } \\
\text { memories }\end{array}$ \\
\hline [32] & body bias technique & 0.35 & 3 & 10 & 6 & - & - & NVM \\
\hline [33] & clocking scheme & 0.18 & 1.8 & 100 & 10.1 & 55 & - & $\begin{array}{l}\text { Flash } \\
\text { memories }\end{array}$ \\
\hline [34] & all PMOS CP & 0.35 & 2 & 3 & 15.5 & - & - & $\begin{array}{l}\text { Memory } \\
\text { application }\end{array}$ \\
\hline [35] & $\begin{array}{l}\text { 4-phase complemen- } \\
\text { tarycharge } \\
\text { pump. }\end{array}$ & 0.18 & 1.8 & 4 & 1.89 & 92.01 & - & $\begin{array}{c}\text { DC- } \\
\text { DCconverter }\end{array}$ \\
\hline [39] & static CTS & 0.35 & 1.5 & 1.5 & 7.5 & - & 20 & $\begin{array}{c}\text { DC- } \\
\text { DCconverters }\end{array}$ \\
\hline [40] & $\begin{array}{l}\text { switched-capacitor- } \\
\text { based }\end{array}$ & 0.35 & 2.9 & 0.25 & 5.5 & 90 & 2454.8 & $\begin{array}{c}\text { DC- } \\
\text { DCconverter }\end{array}$ \\
\hline$[41]$ & $\begin{array}{c}\text { dynamically bias the } \\
\text { CTS's }\end{array}$ & 0.18 & 2 & 0.78 & 9.8 & 54 & 15.75 & NVM \\
\hline [43] & dynamic CTS & 0.35 & 3.3 & 3 & $2.5-5$ & - & 0.6 & NVM \\
\hline$[44]$ & $\begin{array}{l}\text { improved charge } \\
\text { sharing method }\end{array}$ & 0.35 & 3.3 & 5 & 14 & 83.3 & - & $\begin{array}{c}\text { DC- } \\
\text { DCconverter }\end{array}$ \\
\hline [47] & regulated $\mathrm{CP}$ & 0.35 & 2.5 & 30 & 16 & 34 & 69 & NVM \\
\hline$[48]$ & $\begin{array}{l}\text { frequency } \\
\text { modulation }\end{array}$ & 0.18 & 2.8 & 10 & 12.8 & 80 & 0.6 & $\begin{array}{c}\text { DC- } \\
\text { DCconverter }\end{array}$ \\
\hline
\end{tabular}

Therefore, from Table 1, it is being found that many proposed CP topologies are focused on reducing ripple voltage, simple design architectures, lower chip area while generating higher output voltage from a very low power supply voltage. CP circuit design schemes are classified based on voltage-controlled or current controlled approaches. Table 1 shows that all the researchers have presented their design topologies considering and maintaining the design mentioned above parameters while designing the CMOS $\mathrm{CP}$ circuits. Conventionally, any memory devices required comparatively higher output voltage (around $5 \mathrm{~V}$ to $23 \mathrm{~V}$ ) generated from the low power supply voltage (about $1.2 \mathrm{~V}$ to $3.3 \mathrm{~V}$ ) to perform their read/write/erase operations. In this application, a regulated voltage supply is in demand, so selecting a CP scheme that has the option for voltage 
regulation would benefit the researchers. Many topologies managed to overcome the threshold voltage drop, reversion and conduction loss by maintaining the proper transistor sizing. By reducing the transistor's parasitic effects, some have achieved higher pumping efficiency (more than $80 \%$ ) with lower chip areas and reduced ripple voltages.

Besides, $[1,2,8,24,33,34]$ proposed CP design schemes, which are suitable for RFID tag memory, flash memories and EEPROM. It is necessary to produce higher output voltage with low ripple and low power dissipation for EEPROM/flash memories to do the read/write/erase operations. Therefore, it is clear from Table 1 that if anyone wanted to design CP circuit for RFID tag memory, then the CTS based modified CP would be the solution [1]. In this method, the design achieved low power, high efficiency, and low output ripple. If all the transistors are appropriately matched, then the parasitic capacitor's effect can be eliminated, generated from this design. Furthermore, this design reduced the chip layout area. This method has successfully integrated into other devices to form the integrated high voltage generator from a very low power supply voltage for RFID tag EEPROM. Eventually, considering flash memory specification, this $\mathrm{CP}$ circuit can be one solution for future researchers.

It is summarized in Table 1 that, though $[32,41,43,47]$ proposed different schemes for designing $\mathrm{CP}$ circuits, but all of these methods are compatible with NVM. In terms of higher output voltage, [41] can generate the higher output voltage, improved pumping efficiency and lower the threshold voltage. However, this design consumed much power due to the intricate design scheme and the chip layout size is more extensive compared to others. On the other hand, the $\mathrm{CP}$ design scheme proposed by [47] has the advantages of minimized short-circuit current, compact design, small chip area and low power consumption. This design is not compatible with all low power applications. Instead, it is only suitable for floating gate tunneling based transistors like NVM and MEMS devices. In Table 1, it is shown that $[35,39,40,44,48]$ also proposed a CP design method for generating boosted output voltage for DC-DC converters. Based on the comparison as shown in Table 1, it can be suggested that if future researchers wanted to design a $\mathrm{CP}$ circuit for generating a high voltage converter or DC-DC converter, they could easily choose the topology proposed by [48]. This proposed topology managed to generate a higher output voltage from the low power supply voltage and higher pumping efficiency $(80 \%)$ and a low chip area of only $0.6 \mathrm{~mm}^{2}$. Among these schemes, [44] has produced higher output voltage and this design has the benefit of eliminating the reverse charge sharing problem. Table 1 also found that the $\mathrm{CP}$ circuit proposed by [44] has ensured higher pumping efficiency with the compensations with zero $V_{\text {th }}$ MOSFET and CTS's can turn on/off completely. Though this design can reduce feedback current, it necessitates increased power dissipation if the stage number grows along with complex circuitry. This design required a larger chip layout area, which is considered a drawback in the CMOS process.

On the other hand, energy harvesting devices also require a CP circuit to boost voltages to perform the process. This sector does not need much higher voltage compared to memory devices. Thus, the pumping efficiency levels are not that much higher in these topologies than other $\mathrm{CP}$ circuits based on low voltage applications, i.e., RFID transponder memory, NVM, DC-DC converters, PLL devices, etc. Moreover, these topologies have also required extra circuitry, which eventually increases the overall chip area and power dissipation. Table 2 shows the summary of the topologies used recently for $\mathrm{CP}$ circuit design. 
Table 2. Performance comparison of different CP design topologies based on energy harvesting.

\begin{tabular}{|c|c|c|c|c|c|c|c|c|}
\hline Source & Design Topology & $\begin{array}{l}\text { Process } \\
(\mu \mathrm{m})\end{array}$ & $\begin{array}{c}\text { Supply } \\
\text { Voltage VDD } \\
\text { (V) }\end{array}$ & $\begin{array}{l}\text { Frequency } f_{c l k} \\
\text { (MHz) }\end{array}$ & $\begin{array}{l}\text { Output } \\
V_{\text {out }}(\mathrm{V})\end{array}$ & $\begin{array}{c}\text { Pumping } \\
\text { Efficiency (\%) }\end{array}$ & $\begin{array}{c}\text { Die Area } \\
\left(\mathrm{mm}^{2}\right)\end{array}$ & Applications \\
\hline$[50]$ & $\begin{array}{l}\text { boost converter } \\
\text { scheme }\end{array}$ & 0.13 & 0.27 & 0.80 & 1.4 & 58 & 0.42 & $\begin{array}{c}\text { energy harvesting } \\
\text { with PV cell }\end{array}$ \\
\hline$[51]$ & $\begin{array}{l}\text { cross-coupled body } \\
\text { bias }\end{array}$ & 0.18 & 0.32 & 0.45 & 2.04 & 89 & 1376 & energy harvesting \\
\hline [53] & $\begin{array}{c}\text { cross-coupled with } \\
\text { DBB }\end{array}$ & 0.13 & 0.15 & 0.25 & 0.619 & 54 & 66 & $\begin{array}{c}\text { low voltage } \\
\text { energyharvesting }\end{array}$ \\
\hline$[54]$ & $\begin{array}{l}\text { single-clock tree } \\
\text { topology }\end{array}$ & 0.18 & $0.39-0.43$ & $17-23$ & 1 & 70 & 0.48 & $\begin{array}{l}\text { microscale solar } \\
\text { energy harvesting }\end{array}$ \\
\hline [55] & closed loop control & 0.18 & 5 & 0.01 & 16.95 & - & 2.53 & $\begin{array}{l}\text { sensor based } \\
\text { microgyroscope }\end{array}$ \\
\hline
\end{tabular}

Table 2 shows that the $\mathrm{CP}$ circuit scheme designed by [50] generates $3 \times$ higher output voltage than the supply voltage. This design requires less chip area. However, this method is unable to provide higher pumping efficiency. On the other hand, [51] has a fast-pumping rate at $0.1 \mathrm{~ms}$ and reduces leakages with body biasing for PMOS only. However, this design required interleaved inverters and extra stages, increasing the power dissipation. In [53], the method works in very low supply voltage with higher output voltage and higher efficiency. This design has the disadvantage, too, as it required off-chip capacitors and extra complex circuitry for clocking and dynamic bulk-biasing. Instead, the CP topology proposed by [54] has achieved a higher output voltage with a lower chip area. Though the pumping efficiency is not highest than other researchers, future researchers would pick this topology if there is any place for the trade-off between efficiency and implementation cost. Finally, [55] proposed CP scheme has the advantages of linear output voltage, stable voltage regulation, and temperature insensitive output voltage. Still, it lacks lower temperature variations, which is considered the disadvantage of this topology.

Therefore, this review illustrates various $\mathrm{CP}$ design topologies for low power applications, which have recommended the considerations and trade-off between low voltage, low power and higher pumping efficiency to select the optimum CP method. This research also suggested that numerous $\mathrm{CP}$ design schemes be compatible with low power applications, especially in RFID tag memory, DC-DC converters and energy harvesting devices. Hence, this review article would be the future guideline to find a better option and scheme to design a CMOS based $\mathrm{CP}$ circuit by considering the critical performance parameters mentioned above for low power applications.

\section{Conclusions}

In this article, various topologies of designing $\mathrm{CP}$ circuits for low power applications are reviewed based on different design schemes. $\mathrm{CP}^{\prime}$ s essential features, i.e., output voltage gain, power efficiency, die area occupation, handling frequency levels, input supply voltage, CMOS process and the charge pumping capabilities, are compared in detail and based on the reviews, suitable applications for these topologies are also illustrated. The three commonly used applications, such as memory, DC-DC converters and energy harvesting area, are identified from various design methods and their performances are figured out from this review. Therefore, this study provides a useful reference for future researchers to select the appropriate design topology for $\mathrm{CP}$ circuits under technological and operational constraints.

Author Contributions: Conceptualization, L.F.R.; methodology, L.F.R.; software, L.F.R.; validation, L.F.R. and M.M.; formal analysis, L.F.R.; resources, L.F.R.; writing-original draft preparation, L.F.R.; writing-review and editing, L.F.R., and M.M.; visualization, L.F.R.; supervision, L.A.; funding acquisition, L.A., and M.B.M. All authors have read and agreed to the published version of the manuscript.

Funding: This research was funded by Ministry of Higher Education Malaysia and Universiti Kebangsaan Malaysia, grant number TRGS/1/2015/UKM/02/5/2. 
Acknowledgments: The authors would like to express sincere gratitude to the Ministry of Higher Education Malaysia and Universiti Kebangsaan Malaysia (UKM) for all the support regarding this review article

Conflicts of Interest: The authors declare no conflict of interest.

\section{References}

1. Rahman, L.F.; Marufuzzaman, M.; Alam, L.; Sidek, L.M.; Reaz, M.B.I. A low power and low ripple CMOS high voltage generator for RFID transponder EEPROM. PLoS ONE 2020, 15, e0225408. [CrossRef]

2. Rahman, L.F.; Alam, L.; Marufuzzaman, M. Design of a Low Power and High-Efficiency Charge Pump Circuit for RFID Transponder EEPROM. Info. MIDEM 2021, 50, 255-262.

3. Rahman, L.F.; Ariffin, N.B.; Reaz, M.B.I.; Marufuzzaman, M. High Performance CMOS Charge Pumps for Phase-locked Loop. Trans. Electr. Electron. Mater. 2015, 16, 241-249. [CrossRef]

4. Dagan, H.; Shapira, A.; Teman, A.; Mordakhay, A.; Jameson, S.; Pikhay, E.; Fish, A. A low-power low-cost 24 GHz RFID tag with a C-flash based embedded memory. IEEE J. Solid State Circuits 2014, 49, 1942-1957. [CrossRef]

5. Navidi, M.M. Integrated Circuits for Programming Flash Memories in Portable Applications. Ph.D. Thesis, Statler College of Engineering and Mineral Resources, Morgantown, WV, USA, 2018.

6. Rahman, L.F.; Reaz, M.B.I.; Yin, C.C.; Ali, M.A.M.; Marufuzzaman, M. Design of high speed and low offset dynamic latch comparator in $0.18 \mu \mathrm{m}$ CMOS Process. PLoS ONE 2014, 9, e108634. [CrossRef] [PubMed]

7. Rahman, L.F.; Reaz, M.B.I.; Marufuzzaman, M. Design of Low Power and Low Phase Noise Current Starved Oscillator for RFID Tag EEPROM. Info. MIDEM 2019, 49, 19-24.

8. Tanzawa, T.; Tanaka, T.; Takeuchi, K.; Nakamura, H. Circuit techniques for a 1.8-V-only NAND flash memory. IEEE J. Solid State Circuits 2002, 37, 84-89. [CrossRef]

9. Kawahara, T.; Kobayashi, T.; Jyouno, Y.; Saeki, S.I.; Miyamoto, N.; Adachi, T.; Kimura, K. Bit-line clamped sensing multiplex and accurate high voltage generator for quarter-micron flash memories. IEEE J. Solid State Circuits 1996, 31, 1590-1600. [CrossRef]

10. Lu, Y.; Ki, W.H.; Yue, C.P. An NMOS-LDO regulated switched-capacitor DC-DC converter with fast-response adaptive-phase digital control. IEEE Trans. Power Electron. 2015, 31, 1294-1303. [CrossRef]

11. Shen, B.; Bose, S.; Johnston, M.L. A $1.2 \mathrm{~V}-20 \mathrm{~V}$ closed-loop charge pump for high dynamic range photodetector array biasing. IEEE Trans. Circuit Syst. Express Briefs 2018, 66, 327-331. [CrossRef]

12. Ballo, A.; Bottaro, M.; Grasso, A.D.; Palumbo, G. Regulated charge pumps: A comparative study by means of Verilog-AMS Electronics 2020, 9, 998. [CrossRef]

13. Palumbo, G.; Pappalardo, D.; Gaibotti, M. Charge pump circuits: Power consumption optimization-a summary. IEEE Circuits Syst. Mag. 2004, 4, 26-29. [CrossRef]

14. Palumbo, G.; Pappalardo, D. Charge pump circuits: An overview on design strategies and topologies. IEEE Circuits Syst. Mag. 2010, 10, 31-45. [CrossRef]

15. File: Cockcroft Walton Voltage Multiplier Circuit.svg. Available online: https://commons.wikimedia.org/wiki/File:Cockcroft Walton_voltage_multiplier_circuit.svg (accessed on 12 April 2015).

16. Dickson, J.F. On-chip high-voltage generation in MNOS integrated circuits using an improved voltage multiplier technique. IEEE J. Solid State Circuits 1976, 11, 374-378. [CrossRef]

17. Witters, J.S.; Groeseneken, G.; Maes, H.E. Analysis and modeling of on-chip high-voltage generator circuits for use in EEPROM circuits. IEEE J. Solid State Circuits 1989, 24, 1372-1380. [CrossRef]

18. Cataldo, G.D.; Palumbo, G. Optimized design of an Nth order Dickson voltage multiplier. IEEE Trans. Circuits Syst. Fundam. Theory Appl. 1996, 43, 414-418. [CrossRef]

19. Palumbo, G.; Barniol, N.; Bethaoui, M. Improved behavioral and design model of an Nth-order charge pump. IEEE Trans. Circuits Syst. Fundam. Theory Appl. 2000, 47, 264-268. [CrossRef]

20. Tanzawa, T.; Tanaka, T. A dynamic analysis of the Dickson charge pump circuit. IEEE J. Solid State Circuits 1997, 32, 1231-1240. [CrossRef]

21. Wang, C.C.; Wu, J.C. Efficiency improvement in charge pump circuits. IEEE J. Solid State Circuits 1997, 32, 852-860. [CrossRef]

22. Wu, J.T.; Chang, K.L. MOS charge pumps for low-voltage operation. IEEE J. Solid State Circuits 1998, 33, $592-597$.

23. Favrat, P.; Deval, P.; Declercq, M.J. A high-efficiency CMOS voltage doubler. IEEE J. Solid State Circuits 1998, 33, 410-416. [CrossRef]

24. Lauterbach, C.; Weber, W.; Romer, D. Charge sharing concept and new clocking scheme for power efficiency and electromagnetic emission improvement of boosted charge pumps. IEEE J. Solid State Circuits 2000, 35, 719-723. [CrossRef]

25. Palumbo, G.; Pappalardo, D.; Gaibotti, M. Charge-pump circuits: Power-consumption optimization. IEEE Trans. Circuits Syst. Fundam. Theory Appl. 2002, 49, 1535-1542. [CrossRef]

26. Canet, P.; Lalande, F.; Razafindramora, J.; Bouquet, V.; Postel-Pellerin, J.; Bouchakour, R.; Mirabel, J.M. Integrated reliability in EEPROM nonvolatile memory cell design. In Proceedings of the IEEE Computational Systems Bioinformatics Conference, Stanford, CA, USA, 17 November 2004; pp. 66-69.

27. Bowen, M.; Pepper, P.; McPhie, R.; Winter, M. Evaluation of a remote drafting system for regulating sheep access to supplement. Anim. Prod. Sci. 2009, 49, 248-252. [CrossRef] 
28. Cockcroft, J.D.; Walton, E.T. Experiments with high velocity positive ions. (I) Further developments in the method of obtaining high velocity positive ions. Proc. R. Soc. Lond. 1932, 136, 619-630.

29. Pylarinos, L.; Roger, E. Charge Pumps: An Overview; Department of Electrical and Computer Engineering University of Toronto: Toronto, ON, Canada, 2003; p. 7.

30. Umezawa, A.; Atsumi, S.; Kuriyama, M.; Banba, H.; Imamiya, K.I.; Naruke, K.; Tanaka, S. A 5-V-only operation 0.6-mu m flash EEPROM with row decoder scheme in triple-well structure. IEEE J. Solid State Circuits 1992, 27, 1540-1546. [CrossRef]

31. Atsumi, S.; Kuriyama, M.; Umezawa, A.; Banba, H.; Naruke, K.; Yamada, S.; Yoshikawa, K. A 16-Mb flash EEPROM with a new self-data-refresh scheme for a sector erase operation. IEICE Trans. Electron. 1994, 77, 791-799. [CrossRef]

32. Lai, S.Y.; Wang, J.S. A high-efficiency CMOS charge pump circuit. In Proceedings of the IEEE International Symposium on Circuits and Systems Cat. No. 01CH37196, Sydney, NSW, Australia, 6-9 May 2001; Volume 4, pp. 406-409.

33. Pelliconi, R.; Iezzi, D.; Baroni, A.; Pasotti, M.; Rolandi, P.L. Power Efficient Charge Pump in Deep Submicron Standard CMOS Technology. IEEE J. Solid State Circuits 2003, 38, 1068-1071. [CrossRef]

34. Yan, N.; Min, H. A high efficiency all-PMOS charge pump for low-voltage operations. In Proceedings of the 2005 IEEE Asian Solid-State Circuits Conference, Hsinchu, Taiwan, 1-3 November 2005; pp. 361-364.

35. Su, L.; Ma, D. Design and optimization of integrated low-voltage low-power monolithic CMOS charge pumps. In Proceedings of the 2008 International Symposium on Power Electronics, Electrical Drives, Automation and Motion, Ischia, Italy, 11-13 June 2008; pp. $43-48$.

36. Richelli, A.; Colalongo, L.; Tonoli, S.; Kovacs, Z. A 0.2 V-1.2 V converter for power harvesting applications. In Proceedings of the 34th European Solid-State Circuits Conference, Edinburgh, UK, 15-19 September 2008; pp. 406-409.

37. Richelli, A.; Mensi, L.; Colalongo, L.; Kovacs, Z.; Rolandi, P.L. A 1.2 V-5 V high efficiency CMOS charge pump for non-volatile memories. In Proceedings of the 2007 IEEE International Symposium on Circuits and Systems, New Orleans, LA, USA, 27-30 May 2007; pp. 2411-2414.

38. Richelli, A.; Mensi, L.; Colalongo, L.; Rolandi, P.L.; Kovacs-Vajna, Z.M. A 1.2-to-8 V charge-pump with improved power efficiency for non-volatille memories. In Proceedings of the 2007 IEEE International Solid-State Circuits Conference, San Francisco, CA, USA, 11-15 February 2007; Digest of Technical Papers. pp. 522-619.

39. Shiau, M.S.; Hsieh, Z.H.; Hsieh, C.C.; Liu, H.Y.; Liu, D.G. A novel static CTS charge pump with voltage level controller for DC-DC converters. In Proceedings of the 2007 IEEE Conference on Electron Devices and Solid-State Circuits, Tainan, Taiwan, 20-22 December 2007; pp. 481-484.

40. Huang, M.-H.; Hsieh, C.-Y.; Fan, P.-C.; Chen, K.-H. A dual-phase charge pump circuit with compact size. Analog. Integr. Circuits Signal Process. 2009, 64, 55-67. [CrossRef]

41. Peng, F.; Yunlong, L.; Nanjian, W. A high efficiency charge pump circuit for low power applications. J. Semicond. 2010, 31, 015009. [CrossRef]

42. Wu, J.T.; Chang, Y.H.; Chang, K.L. 1.2 V CMOS switched-capacitor circuits. In Proceedings of the 1996 IEEE International Solid-State Circuits Conference, San Francisco, CA, USA, 10 February 1996; Digest of TEchnical Papers. pp. 388-389.

43. Dong-Sheng, L.; Xue-Cheng, Z.; Fan, Z.; Min, D. Embeded EEPROM memory achieving lower power-new design of EEPROM memory for RFID tag IC. IEEE Circuits Devices Mag. 2006, 22, 53-59. [CrossRef]

44. Yan, L.; Shilin, Z.; Yiqiang, Z. High voltage generator circuit with low power and high efficiency applied in EEPROM. J. Semicond. 2012, 33, 065006.

45. Wei, K.C.; Amin, M.S.; Reaz, B. Low voltage charge pump circuit using $0.18 \mu \mathrm{m}$ CMOS technology. Rev. Roum. Sci. Techn. Électrotechn. Énerg. 2013, 58, 83-92.

46. Zucchelli, M.; Colalongo, L.; Richelli, A.; Kovacs-Vajna, Z.M. Dickson charge pump using integrated inductors in complementary metal-oxide semiconductor technology. IET Power Electron. 2016, 9, 553-558. [CrossRef]

47. Rumberg, B.; Graham, D.W.; Navidi, M.M. A regulated charge pump for tunneling floating-gate transistors. IEEE Trans. Circuit Syst. Express Briefs 2017, 64, 516-527. [CrossRef]

48. Abdi, A.; Kim, H.S.; Cha, H.K. A High-Voltage Generation Charge-Pump IC using Input Voltage Modulated Regulation for Neural Implant Devices. IEEE Trans. Circuit Syst. Express Briefs 2018, 66, 342-346. [CrossRef]

49. Richelli, A.; Colalongo, L.; Mensi, L.; Cacciatori, A.; Kovacs-Vajna, Z.M. Charge pump architectures based on dynamic gate control of the pass-transistors. IEEE Trans. Very Large Scale Integr. VLSI Syst. 2009, 17, 964-967. [CrossRef]

50. Shih, Y.C.; Otis, B.P. An Inductor less DC-DC Converter for Energy Harvesting With a $1.2 \mu W$ Bandgap-Referenced Output Controller. IEEE Trans. Circuit Syst. Express Briefs 2011, 58, 832-836. [CrossRef]

51. Peng, H.; Tang, N.; Yang, Y.; Heo, D. CMOS start-up charge pump with body bias and backward control for energy harvesting step-up converters. IEEE Trans Circuits Syst. Regul. Pap. 2014, 61, 1618-1628. [CrossRef]

52. Ker, M.D.; Chen, S.L. Ultra-high-voltage charge pump circuit in low-voltage bulk CMOS processes with polysilicon diodes. IEEE Trans. Circuit Syst. Express Briefs 2007, 54, 47-51. [CrossRef]

53. Kim, J.; Mok, P.K.; Kim, C. A 0.15 V input energy harvesting charge pump with dynamic body biasing and adaptive dead-time for efficiency improvement. IEEE J. Solid State Circuits 2015, 50, 414-425. [CrossRef]

54. Mondal, S.; Paily, R. An efficient on-chip switched-capacitor-based power converter for a microscale energy transducer. IEEE Trans. Circuit Syst. Express Briefs 2015, 63, 254-258. [CrossRef]

55. Li, X.; Li, R.; Ju, C.; Hou, B.; Wei, Q.; Zhou, B.; Zhang, R. A Regulated Temperature-Insensitive High-Voltage Charge Pump in Standard CMOS Process for Micromachined Gyroscopes. Sensors 2019, 19, 4149. [CrossRef] [PubMed] 